\title{
Antioxidant activity of myricetin-like compounds
}

Gabriel L. C. de Souza ${ }^{1 *}$, Shawan K. C. Almeida ${ }^{1}$, and Rodrigo A. Mendes ${ }^{1}$ gabriellcs@pq.cnpq.br

${ }^{1}$ Departamento de Química, Universidade Federal de Mato Grosso, 78060-900 Cuiabá, Brazil

Keywords: antioxidant activity, myricetin-like compounds, bond dissociation energy, ionization potential.

It is well known that the antioxidant potential of flavonols can be probed through the determination of the bond dissociation enthalpies (BDEs) for $\mathrm{OH}$ groups and ionization potentials (IPs), related to the hydrogen-atom transfer mechanism (HAT) and single electron transfer mechanism (SET), respectively ${ }^{1}$.

In this work, the structures and energetics of myricetin 4'-O-alpha-Lrhamnopyranoside and myricetin 3-O-alpha-L-rhamnopyranoside (see Figure 1 for structures) were examined with density functional theory, using B3LYP ${ }^{2}$ and LC$\omega \mathrm{PBE}^{3}$ functionals with both the $6-311 \mathrm{G}(\mathrm{d}, \mathrm{p})$ and $6-311+\mathrm{G}(\mathrm{d}, \mathrm{p})$ basis sets ${ }^{4}$. In addition, BDEs and IPs were determined in gas-phase, water, and methanol. The computations were performed using the Gaussian 09 software suite ${ }^{5}$.

(a)

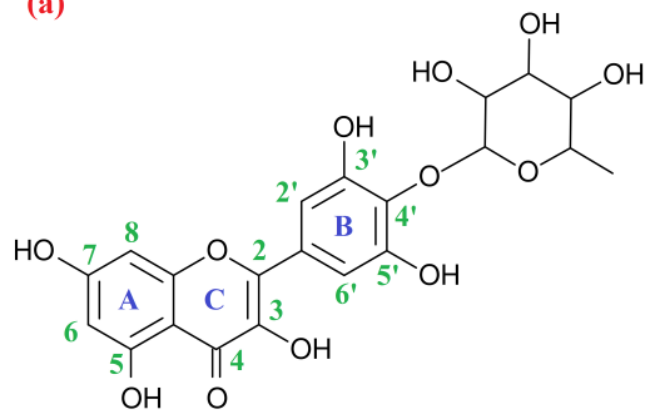

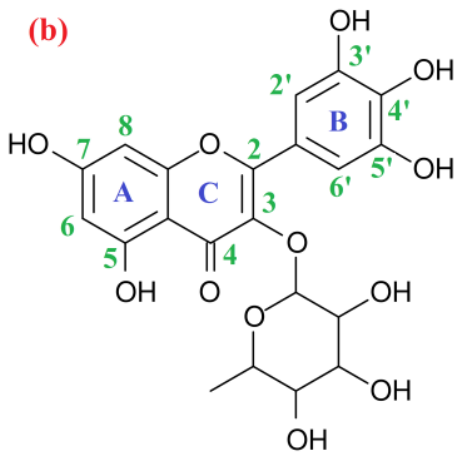

(b)

$\mathrm{OH}$

Figure 1. Chemical structures: (a) myricetin 4'-O-alpha-L-rhamnopyranoside and (b) myricetin 3-Oalpha-L-rhamnopyranoside.

The smallest BDEs among the five $\mathrm{OH}$ groups of each compound were determined (using LC- $\omega$ PBE/6-311G(d,p) in water, for instance) to be $79.54 \mathrm{kcal} / \mathrm{mol}$ for myricetin 4'-O-alpha-L-rhamnopyranoside and $75.21 \mathrm{kcal} / \mathrm{mol}$ for myricetin 3-Oalpha-L-rhamnoside while the IPs (at the same level of theory) were obtained as 142.56 and $141.27 \mathrm{kcal} / \mathrm{mol}$, respectively. By comparing with known antioxidants, these values for the BDEs indicate both compounds show promise for antioxidant activity, especially myricetin 3-O-alpha-L-rhamnopyranoside. In addition, the position of the sugar moiety in myricetin has a moderate effect on the BDEs for all $\mathrm{OH}$ groups. Interestingly, the BDEs suggest that (depending on the position of inclusion) the sugar moiety can lead to an increase in the antioxidant activity. Therefore, further experimental tests are encouraged. Additional results will be presented and discussed during the Conference.

\section{Acknowledgments}

This work has been supported by CNPq.

\section{References}

[1] J. S. Wright et al., J. Am. Chem. Soc. 123, 1173 (2001).

[2] A. D. Becke, J. Chem. Phys. 98, 5648 (1993).

[3] O. A. Vydrov and G. E. Scuseria, J. Chem. Phys. 125, 234109 (2006).

[4] M. J. Frisch, J. A. Pople and J. S. Binkley, J. Chem. Phys. 80, 3265 (1984).

[5] M. J. Frisch et al., Gaussian Inc., Wallingford CT, G09, Revision D.01 (2009). 